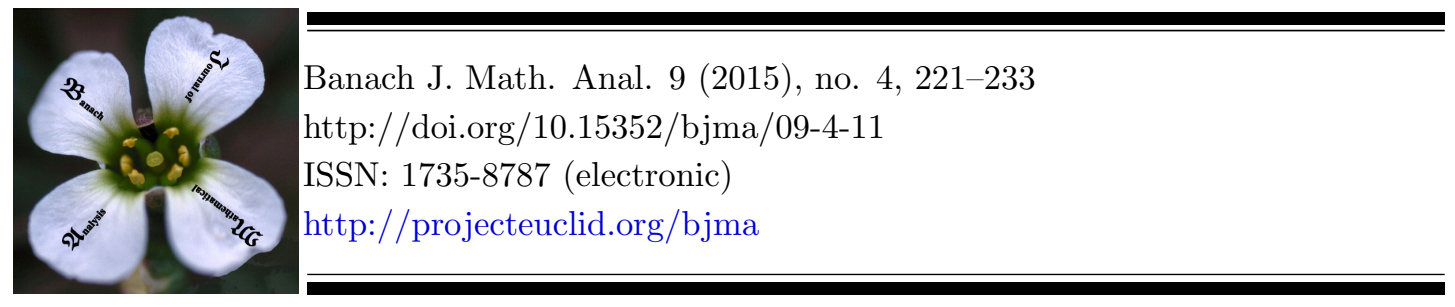

\title{
ALGEBRAIC AND TOPOLOGICAL PROPERTIES OF THE GROUP OF ISOMETRIES ON CLASSES OF VECTOR VALUED FUNCTION SPACES
}

\author{
FERNANDA BOTELHO* AND JAMES JAMISON \\ Communicated by J. A. Ball
}

\begin{abstract}
We study algebraic and topological properties of the group of all surjective isometries on several spaces of vector valued analytic functions and vector valued $L^{p}$ spaces $(1 \leq p \leq \infty)$. We also derive the form for the surjective linear isometries on the vector valued little Zygmund space.
\end{abstract}

\section{INTRODUCTION}

Let $E$ be a Banach space and let $G(E)$ be the group of surjective linear isometries of $E$. Under the standard composition of operators and the strong operator topology, the isometry group $G(E)$ is a topological group.

The problem of describing all the surjective linear isometries supported by a given Banach space goes back to Banach. In [3], it was shown that surjective linear isometries on spaces of continuous functions defined on a compact metric space are weighted composition operators. Since Banach's initial work, the isometry group has been completely characterized for many Banach spaces. For a survey of these results we refer the reader to the books [12] and the references therein. It is a consequence of a result by K. Jarosz [18] that every Banach space can be equivalently renormed so that the isometry group is isomorphic to $\mathbb{S}^{1}$. Further there are extremely non complex Banach spaces whose isometry group is a discrete Boolean group, see [17] and also [16].

Date: Received: Sep. 23, 2014; Accepted: Jan. 26, 2015.

* Corresponding author.

2010 Mathematics Subject Classification. Primary 47B38; Secondary 47B48.

Key words and phrases. Surjective isometry, isometry invariance, invariant subspace, function space. 
Isometries on a given function space, for which a characterization is known, are often described in terms of a list "symbols". As for example, a surjective linear isometry $T$, on $\mathcal{C}(\Omega)$, the space of continuous functions defined on a compact metric space $\Omega$, is of the form

$$
T(f)=\lambda f \circ \varphi, \quad \forall f \in \mathcal{C}(\Omega),
$$

with $\lambda: \Omega \rightarrow \mathbb{S}^{1}$ continuous and $\varphi$ a homeomorphism of $\Omega$. We refer to $\lambda$ and $\varphi$ as the symbols associated with $T$. This fact determines an algebraic decomposition of the isometry group of certain classes of function spaces in terms of the isometry groups of the respective symbols. This motivates the main ideas addressed in this paper. More precisely, we study the algebraic structure of the isometry groups for several vector valued function spaces and from that draw conclusions about topological properties of those groups.

These questions were previously considered by Berkson and Porta for the isometry group of $H^{p}(\triangle)$, where $\triangle$ denotes the open unit disc in the complex plane. In [5], it is shown that $G\left(H^{p}(\triangle)\right) \nsubseteq \mathbb{S}^{1} \times A u t(\triangle)$, for $1<p<\infty$ but $G\left(H^{1}(\triangle)\right) \cong \mathbb{S}^{1} \times A u t(\triangle)$. Arazy, in [1], characterizes the isometries of the symmetric sequence spaces $E$ and observes that $G(E) \cong G_{0} \rtimes \mathcal{P}$, the semi-direct product of $G_{0}$ ( the group of all diagonal multiplications by modulus 1 complex numbers) with $\mathcal{P}$ ( the group of all isometries defined via the action of a permutation). Recently, in [14], Gardella and Thiel have derived a similar decomposition for the standard Lebesgue space, $L^{p}$ with $p \in[1, \infty) \backslash\{2\}$. More precisely, $G\left(L^{p}(\Omega, \mu) \cong A u t_{*}(\Omega) \rtimes L^{0}\left(\Omega, \mathbb{S}^{1}\right)\right.$, where $L^{0}\left(\Omega, \mathbb{S}^{1}\right)$ is the Abelian group (under pointwise multiplication) of all measurable functions $\Omega \rightarrow \mathbb{S}^{1}$ and $A u t_{*}(\Omega)$ is the group (under composition) of regular set isomorphisms on the $\sigma$-algebra of $\mu$-measurable subsets of $\Omega$.

In this paper we investigate algebraic and topological properties of the group of all isometries for vector valued versions of Hardy spaces, Novinger-Oberlin spaces, little Bloch and Zygmund spaces and classes of $L^{p}$ spaces. For all these spaces, the form for the surjective isometries leads to a natural decomposition of the group of all isometries which implies some topological properties of those groups.

Further, we also extend a characterization of the surjective isometries on the scalar valued little Zygmund space, presented in [7], to the vector valued little Zygmund space with the range space satisfying some additional properties. This is obtained by transferring a surjective isometry on the little Zygmund space to the little Bloch space and then employ a characterization of the surjective isometries on the latter derived in [8].

Throughout this paper we use the notation $G=H \rtimes K$ to denote the semidirect product of the subgroups of $G, H$ and $K$ where $H$ is a normal subgroup of $G$. Since we will appeal to [11, Theorem 12 (p. 180)] in many occasions to follow, we state this theorem for an easier reading.

Theorem. (cf. [11]) Suppose $G$ is a group with subgroups $H$ and $K$ such that $H$ is a normal subgroup of $G$ and $H \cap K=1$. Then $H K$ is isomorphic to $H \rtimes K$, $(H K \cong H \rtimes K)$. In particular, if $G=H K$ then $G$ is the semidirect product of 
$H$ and $K, G \cong H \rtimes K$.

We also set some notation to be used in this paper. We recall that the symbol $\triangle$ denotes the unit open disc in the complex plane and the group of disc automorphisms, Aut $(\triangle)$, is the set of all $\sigma: \triangle \rightarrow \triangle$ given by $\sigma(z)=\lambda \frac{z-z_{0}}{1-z_{0} z}$ with $z_{0} \in \triangle$ and $\lambda$ a modulus 1 complex number.

\section{Algebraic and Topological Structure of the Isometry Group OF $H_{\mathcal{H}}^{1}(\triangle)$}

Let $\mathcal{H}$ be a separable and complex Hilbert space with inner product $\langle\cdot, \cdot\rangle$ and induced norm $\|\cdot\|_{\mathcal{H}}$. We recall that a function $f: \triangle \rightarrow \mathcal{H}$ is analytic if and only if for every $\mathbf{w} \in \mathcal{H}$, the complex valued function $z \rightarrow\langle f(z), \mathbf{w}\rangle$ is analytic. The set

$$
\left\{f: \triangle \rightarrow \mathcal{H} \text { s.t. } f \text { is analytic and } \sup _{0<r<1} \int_{0}^{2 \pi}\left\|f\left(r e^{i \theta}\right)\right\|_{\mathcal{H}} d \theta \text { is finite }\right\}
$$

endowed with the norm $\|f\|_{H_{\mathcal{H}}^{1}(\triangle)}=\frac{1}{2 \pi} \sup _{0<r<1} \int_{0}^{2 \pi}\left\|f\left(r e^{i \theta}\right)\right\|_{\mathcal{H}} d \theta$, is a Banach space denoted by $H_{\mathcal{H}}^{1}(\triangle)$.

Theorem 2.1. (cf. [10]) $T: H_{\mathcal{H}}^{1}(\triangle) \rightarrow H_{\mathcal{H}}^{1}(\triangle)$ is a surjective linear isometry if and only if there exists a unitary operator $U$ and a disc automorphism $\varphi$ such that for every $f \in H_{\mathcal{H}}^{1}(\triangle)$ and $z \in \triangle$

$$
(T f)(z)=U \varphi^{\prime}(z) f(\varphi(z)) .
$$

In the following lemma $\mathcal{U}(\mathcal{H})$ denotes the unitary group on $\mathcal{H}$.

Lemma 2.2. $G\left(H_{\mathcal{H}}^{1}(\triangle)\right)$ is isomorphic to $\mathcal{U}(\mathcal{H}) \times A u t(\triangle)$.

Proof. We define $\Phi: G\left(H_{\mathcal{H}}^{1}(\triangle)\right) \rightarrow \mathcal{U}(\mathcal{H}) \times \operatorname{Aut}(\triangle)$ as follows $\Phi(T)=\left(U_{T}, \varphi_{T}^{-1}\right)$ where $U_{T}$ and $\varphi_{T}$ are the symbols associated with the isometry $T$ as in Theorem 2.1. We first show that $\Phi$ is well-defined, in other words the representation described in Theorem 2.1 is unique. Towards this, we assume that there exists a unitary operator $V_{T}$ and a disc automorphism $\psi_{T}$ such that, for every $f \in H_{\mathcal{H}}^{1}(\triangle)$ and $z \in \triangle$,

$$
U_{T} \varphi_{T}^{\prime}(z) f\left(\varphi_{T}(z)\right)=V_{T} \psi_{T}^{\prime}(z) f\left(\psi_{T}(z)\right) .
$$

This equation applied first to a constant function equal to $\mathbf{v}$, a unit vector in $\mathcal{H}$, and then to $f(z)=z \mathbf{v}$ yields

$$
\varphi_{T}^{\prime}(z) U_{T} \mathbf{v}=\psi_{T}^{\prime}(z) V_{T} \mathbf{v} \text { and } \varphi_{T}^{\prime}(z) \varphi_{T}(z) U_{T} \mathbf{v}=\psi_{T}^{\prime}(z) \psi_{T}(z) V_{T} \mathbf{v}
$$

These equations imply that $\varphi_{T}=\psi_{T}$ and $U_{T}=V_{T}$. It is clear that $\Phi$ is bijective. We now show that $\Phi$ is a homomorphism. Given $T$ and $S$ isometries, then $\Phi(T S)=\left(U_{T S}, \varphi_{T S}^{-1}\right)$ and

$$
T S f(z)=U_{T} U_{S}\left(\varphi_{S} \circ \varphi_{T}\right)^{\prime}(z) f\left(\varphi_{S} \circ \varphi_{T}(z)\right) .
$$

The uniqueness of the representation in (2.1) applied to $T S$ implies that $U_{T S}=$ $U_{T} U_{S}$ and $\varphi_{T S}^{-1}=\left(\varphi_{S} \circ \varphi_{T}\right)^{-1}=\varphi_{T}^{-1} \circ \varphi_{S}^{-1}$. This completes the proof. 
Remark 2.3. The previous result defines two natural subgroups of the isometry group: the subgroup isomorphic to $\mathcal{U}(\mathcal{H})$ consisting of isometries of the form $f \rightarrow U f$ with $U$ a unitary operator on $\mathcal{H}$ and the subgroup isomorphic to $\operatorname{Aut}(\triangle)$, consisting of isometries of the form $f \rightarrow f \circ \varphi$ with $\varphi \in \operatorname{Aut}(\triangle)$.

There are many more subgroups of the isometry group that can be associated with specific subgroups of $A u t(\triangle)$. For example,

(1) The subgroup consisting of two elements, the identity and a composition operator with a disc automorphism whose square is equal to the identity, this means a disc automorphism of the form $\varphi(z)=\frac{z_{0}-z}{1-\overline{z_{0}} z}$, for a given $\left|z_{0}\right|<1$.

(2) The subgroup consisting of composition operators $f \rightarrow f \circ \varphi$ with $\varphi$ a parabolic automorphisms with a given fixed point $\alpha$ of modulus 1 . These are automorphisms of the form, $\varphi(z)=\frac{(1-i c) z+i c \alpha}{-i c \bar{\alpha} z+1+i c}$, with $c \in \mathbb{R}$. This collection of automorphisms define a subgroup of $\operatorname{Aut}(\triangle)$.

It is also straightforward to establish the existence of finite cyclic subgroups of $A u t(\triangle)$ but a complete classification of all subgroups of $A u t(\triangle)$ seems to be unknown.

We consider the isometry group of $H_{\mathcal{H}}^{1}(\triangle)$ and of $\mathcal{U}(\mathcal{H})$ both endowed with the strong operator topology, and $\operatorname{Aut}(\triangle)$ with the uniform topology.

In the proof given for Lemma 2.2 we consider the group isomorphism $\Phi$ : $G\left(H_{\mathcal{H}}^{1}(\triangle)\right) \rightarrow \mathcal{U}(\mathcal{H}) \times A u t(\triangle)$ defined by $\Phi(T)=\left(U_{T}, \varphi_{T}^{-1}\right)$. The next result shows that $\Phi$ is a homeomorphism.

Lemma 2.4. $G\left(H_{\mathcal{H}}^{1}(\triangle)\right)$ is homeomorphic to $\mathcal{U}(\mathcal{H}) \times A u t(\triangle)$.

Proof. We show that $\Phi$ and $\Phi^{-1}$ are continuous maps. Since $\Phi$ is a group homomorphism it is sufficient to show the continuity of $\Phi$ at $I d$. Towards this we consider a sequence of operators $T_{n}$ converging to the identity, which implies that for every $f,\left\|\left(T_{n}-I\right) f\right\|_{H_{\mathcal{H}}^{1}(\triangle)} \rightarrow 0$, with

$$
\begin{aligned}
\left\|T_{n} f-f\right\|_{\mathbf{H}_{\mathcal{H}}^{1}(\triangle)} & =\frac{1}{2 \pi} \sup _{0<r<1} \int_{0}^{2 \pi}\left\|T_{n} f\left(r e^{i \theta}\right)-f\left(r e^{i \theta}\right)\right\|_{\mathcal{H}} d \theta \\
& =\frac{1}{2 \pi} \sup _{0<r<1} \int_{0}^{2 \pi}\left\|\varphi_{n}^{\prime}\left(r e^{i \theta}\right) U_{n} f \circ \varphi_{n}\left(r e^{i \theta}\right)-f\left(r e^{i \theta}\right)\right\|_{\mathcal{H}} d \theta,
\end{aligned}
$$

with $U_{n} \in \mathcal{U}(\mathcal{H})$ and $\varphi_{n} \in \operatorname{Aut}(\triangle)$.

For a fixed $r$ and for every $f$, we have that $g_{n}(\theta)=\| \varphi_{n}^{\prime}\left(r e^{i \theta}\right) U_{n} f \circ \varphi_{n}\left(r e^{i \theta}\right)-$ $f\left(r e^{i \theta}\right) \|_{\mathcal{H}}$ converges to 0 in measure. Then there exists a subsequence of $\left\{g_{n}\right\}$, $\left\{g_{n_{k}}\right\}_{n}$ converges to 0 almost everywhere. For simplicity of notation, we assume that

$$
\lim _{n}\left\|\varphi_{n}^{\prime}\left(r e^{i \theta}\right) U_{n} f \circ \varphi_{n}\left(r e^{i \theta}\right)-f\left(r e^{i \theta}\right)\right\|_{\mathcal{H}}=0
$$

almost everywhere. In particular, for $f$ a constant function equal $\mathbf{v}$, a unit vector in $\mathcal{H}$, we have that $\left\|\varphi_{n}^{\prime}\left(r e^{i \theta}\right) U_{n} \mathbf{v}-\mathbf{v}\right\|_{\mathcal{H}}$ converges to zero almost everywhere. Each disc automorphism $\varphi_{n}$ is of the form $\varphi_{n}(z)=\lambda_{n} \frac{z-z_{n}}{1-\overline{z_{n}} z}$, with $\left|\lambda_{n}\right|=1$ and 
$\left|z_{n}\right|<1$. Thus $\varphi_{n}^{\prime}(z)=\lambda_{n} \frac{1-\left|z_{n}\right|^{2}}{\left(1-\overline{z_{n}} z\right)^{2}}$, and

$$
\lim _{n}\left\|\lambda_{n} \frac{1-\left|z_{n}\right|^{2}}{\left(1-\overline{z_{n}} z\right)^{2}} U_{n} \mathbf{v}-\mathbf{v}\right\|_{\mathcal{H}}=0 \text { a.e. }
$$

implies that $\theta \rightarrow \frac{1-\left|z_{n}\right|^{2}}{\left(1-\overline{z_{n}} r e^{i \theta}\right)^{2}}$ converges to 1 a.e. . This follows from the inequality

$$
|| \lambda_{n}\left|\frac{1-\left|z_{n}\right|^{2}}{\left|1-\overline{z_{n}} z\right|^{2}}\left\|U_{n} \mathbf{v}\right\|_{\mathcal{H}}-\|\mathbf{v}\|_{\mathcal{H}}\right| \leq\left\|\lambda_{n} \frac{1-\left|z_{n}\right|^{2}}{\left(1-\overline{z_{n}} z\right)^{2}} U_{n} \mathbf{v}-\mathbf{v}\right\|_{\mathcal{H}} .
$$

We select a subsequence such that $z_{n_{k}} \rightarrow \rho_{0} e^{i \theta_{0}}$ and $\lambda_{n_{k}} \rightarrow \lambda_{0}$. We observe that $\rho_{0}<1$ and $1-\rho_{0}^{2}=1-2 \rho_{0} r \cos \left(\theta-\theta_{0}\right)+\rho_{0}^{2} r^{2}$ a.e.. Since this equality holds for a sequence of values $\theta$ such that $\theta-\theta_{0}$ converges to $\pi / 2$, we have that $\rho_{0}=0$. Similar considerations applied to $f(z)=z \mathbf{v}$ imply

$$
\lim _{n}\left\|\varphi_{n}^{\prime}\left(r e^{i \theta}\right) \varphi_{n}\left(r e^{i \theta}\right) U_{n} \mathbf{v}-r e^{i \theta} \mathbf{v}\right\|_{\mathcal{H}}=0, \text { a.e.. }
$$

We have the following inequality

$$
\begin{aligned}
|| \varphi_{n}\left(r e^{i \theta}\right)-r e^{i \theta} \mid & \left|\varphi_{n}^{\prime}\left(r e^{i \theta}\right)\right|-r\left\|\varphi_{n}^{\prime}\left(r e^{i \theta}\right) U_{n} \mathbf{v}-\mathbf{v}\right\|_{\mathcal{H}} \mid \leq \\
& \left.\|\left[\varphi_{n}\left(r e^{i \theta}\right)-r e^{i \theta}\right] \varphi_{n}^{\prime}\left(r e^{i \theta}\right) U_{n} \mathbf{v}+r e^{i \theta} \varphi_{n}^{\prime}\left(r e^{i \theta}\right) U_{n} \mathbf{v}-\mathbf{v}\right] \|_{\mathcal{H}}= \\
& \left\|\left[\varphi_{n}\left(r e^{i \theta}\right) \varphi_{n}^{\prime}\left(r e^{i \theta}\right) U_{n} \mathbf{v}-\mathbf{v}\right]\right\|_{\mathcal{H}} .
\end{aligned}
$$

For almost every $\theta$ in the interval $[0,2 \pi]$, we have

$$
\lim _{n} \varphi_{n}\left(r e^{i \theta}\right)=r e^{i \theta}
$$

This implies that $\varphi_{n}$ converges to the identity on $\triangle$ and $\lambda_{0}=1$. We now show that $U_{n}$ converges strongly to the $I d_{\mathcal{H}}$. It was shown before that

$$
\left\|\varphi_{n}^{\prime}\left(r e^{i \theta}\right) U_{n} \mathbf{v}-\mathbf{v}\right\|_{\mathcal{H}} \rightarrow 0 \text {, a.e. . }
$$

Since

$$
\left\|U_{n} \mathbf{v}-\mathbf{v}\right\|_{\mathcal{H}} \leq\left\|\varphi_{n}^{\prime}\left(r e^{i \theta}\right) U_{n} \mathbf{v}-\mathbf{v}\right\|_{\mathcal{H}}+\left|\varphi_{n}^{\prime}\left(r e^{i \theta}\right)-1\right|
$$

we have that $\left\|U_{n} \mathbf{v}-\mathbf{v}\right\|_{\mathcal{H}} \rightarrow 0$ and $\varphi_{n}^{\prime}\left(r e^{i \theta}\right) \rightarrow 1$. This completes the proof.

Theorem 2.5. The isometry group of $H_{\mathcal{H}}^{1}(\triangle)$ is path connected.

Proof. We show that $\mathcal{U}(\mathcal{H}) \times A u t(\triangle)$ is path connected. Given two disc automorphisms $\varphi_{0}(z)=\lambda_{0} \frac{z-\rho_{0} e^{i \theta_{0}}}{1-\rho_{0} e^{-i \theta_{0} z}}$ and $\varphi_{1}=\lambda_{1} \frac{z-\rho_{1} e^{i \theta_{1}}}{1-\rho_{1} e^{-i \theta_{1} z}}$, with $\lambda_{i}=e^{i \alpha_{i}}$ with $i=0,1$. Then

$$
H_{t}(z)=e^{i\left(t \alpha_{0}+(1-t) \alpha_{1}\right)} \frac{z-\left(t \rho_{0}+(1-t) \rho_{1}\right) e^{i\left(t \theta_{0}+(1-t) \theta_{1}\right.}}{1-\left(t \rho_{0}+(1-t) \rho_{1}\right) e^{-i\left(t \theta_{0}+(1-t) \theta_{1} z\right.}}
$$

defines a path of disc automorphisms such that $H_{0}=\varphi_{1}$ and $H_{1}=\varphi_{0}$. We show that every unitary operator $U$ can be connected to the $I d$ by a path of unitary operators. Given $U$, a unitary operator, by the Spectral Theorem for normal operators, there exists of a spectral measure $M(\mu)$ relative to which $U$ has the representation

$$
U=\int_{0}^{2 \pi} e^{i \mu} d M(\mu)
$$


Therefore $H_{t}=\int_{0}^{2 \pi} e^{i t \mu} d M(\mu)$ defines a path of unitary operators on $\mathcal{H}$ such that $H_{0}=I d$ and $H_{1}=U$. Since $\mathcal{U}(\mathcal{H})$ and $A u t(\triangle)$ are path connected the proof is complete.

We observe that $G\left(H_{\mathcal{H}}^{1}(\triangle)\right)$ is a Lie group and if the range space is finite dimensional, say of dimension equal to $n$, the corresponding Lie algebra has dimension $n+1$.

We now state a characterization due to Pei-Kee Lin for surjective isometries on $H^{\infty}(E)$, the set of all $E$-valued bounded analytic functions defined on the open unit disc $\triangle$ with the sup norm. This result extends an earlier result due to M. Cambern with $E$ a finite-dimensional Hilbert space or finite-dimensional complex Banach spaces, cf. [9].

Theorem 2.6. (cf. [19]) Let $E$ be a uniformly smooth and uniformly convex Banach space. Then $T$ is a surjective linear isometry of $H^{\infty}(E)$ if and only if there exist an isometry $U$ from $E$ onto $E$ and an automorphism $\varphi$ of the unit disc onto itself such that $(T f)(z)=U\left(f(\varphi(z))\right.$, for every $f \in H^{\infty}(E)$ and $z \in \triangle$.

By similar techniques to those followed in this section we can also state the following corollary.

Corollary 2.7. If $E$ is a uniformly smooth and uniformly convex Banach space then $G\left(H^{\infty}(E)\right)$ is isomorphic to $\mathcal{U}(E) \times \operatorname{Aut}(\triangle)$.

Forelli in [13] has characterized the isometries on the scalar valued $H^{p}(1<$ $p<\infty$ and $p \neq 2$ ). In [20], $\mathrm{P}-\mathrm{K}$ Lin generalized this result to surjective isometries on $H^{p}(E)(1<p<\infty$ and $p \neq 2)$ with $E$ a complex Hilbert space. Lin's Theorem states that given a surjective isometry $T$ on $H^{p}(E)$ there exist a disc automorphism $\varphi$ and a surjective isometry $U$ on $E$ such that $T(f)(z)=$ $\left(\varphi^{\prime}(z)\right)^{1 / p} U(f(\varphi(z)))$, for all $f \in H^{p}(E)$ and $z \in \triangle$. Since there are several $p$-roots of 1 , it is easy to see that the uniqueness of the representation for a surjective isometry on this setting does not hold. This fact has been mentioned by Berkson and Porta in [5] for the scalar valued $H^{p}$.

\section{Algebraic and Topological Structure of the Isometry Group of Novinger-OBERLIN SPACES}

The Novinger-Oberlin space, $\mathcal{S}_{\mathcal{H}}(\triangle)$, consists of all analytic functions $f$ defined on the open unit disc with values in a complex separable Hilbert space $\mathcal{H}$ and derivative $f^{\prime} \in H_{\mathcal{H}}^{1}(\triangle)$. This space is endowed with the following norm: $\|f\|=$ $\|f(0)\|_{\mathcal{H}}+\left\|f^{\prime}\right\|_{H_{\mathcal{H}}^{1}}$. These spaces were studied in [15] by Hornor and Jamison, where the following characterization for the surjective isometries is given.

Theorem 3.1. (cf. [15]) Let $\mathcal{H}$ be a complex separable Hilbert space. Then $T: \mathcal{S}_{\mathcal{H}}(\triangle) \rightarrow \mathcal{S}_{\mathcal{H}}(\triangle)$ is a surjective linear isometry if and only if there exist $U$ and $V$ surjective linear isometries on $\mathcal{H}$ and $\varphi$ and disc automorphism such that

$$
T f(z)=U f(0)+V[(f \circ \varphi)(z)-(f \circ \varphi)(0)]
$$

for every $f \in \mathcal{S}_{\mathcal{H}}(\triangle)$ and $z \in \triangle$. 
Theorem 3.2. $G\left(\mathcal{S}_{\mathcal{H}}(\triangle)\right)$ is isomorphic to $\mathcal{U}(\mathcal{H}) \times \mathcal{U}(\mathcal{H}) \times \operatorname{Aut}(\triangle)$.

Proof. Given a surjective isometry $T$ there exist surjective isometries $U_{T}$ and $V_{T}$ on $\mathcal{H}$ and $\varphi_{T}$ a disc automorphism such that

$$
T f(z)=U_{T} f(0)+V_{T}\left[\left(f \circ \varphi_{T}\right)(z)-\left(f \circ \varphi_{T}\right)(0)\right]
$$

for every $f \in \mathcal{S}_{\mathcal{H}}(\triangle)$ and $z \in \triangle$. Then we define $\Phi: G\left(\mathcal{S}_{\mathcal{H}}(\triangle)\right) \rightarrow \mathcal{U}(\mathcal{H}) \times$ $\mathcal{U}(\mathcal{H}) \times$ Aut $(\triangle)$ given by $\Phi(T)=\left(U_{T}, V_{T}, \varphi_{T}^{-1}\right)$. We show that $\Phi$ is well defined. We assume that a surjective isometry $T$ has two representations, so that

$$
U_{T} f(0)+V_{T}\left[\left(f \circ \varphi_{T}\right)(z)-\left(f \circ \varphi_{T}\right)(0)\right]=U_{T}^{\prime} f(0)+V_{T}^{\prime}\left[\left(f \circ \psi_{T}\right)(z)-\left(f \circ \psi_{T}\right)(0)\right],
$$

for every $f \in \mathcal{S}_{\mathcal{H}}(\triangle)$ and $z \in \triangle$. In particular, given a unit vector $\mathbf{v}$, (3.1) applied to the constant function $f_{0}=\mathbf{v}$ yields $U_{T} \mathbf{v}=U_{T}^{\prime} \mathbf{v}$, then $U_{T}=U_{T}^{\prime}$. Therefore (3.1) simplifies to

$$
V_{T}\left[\left(f \circ \varphi_{T}\right)(z)-\left(f \circ \varphi_{T}\right)(0)\right]=V_{T}^{\prime}\left[\left(f \circ \psi_{T}\right)(z)-\left(f \circ \psi_{T}\right)(0)\right] .
$$

This equation applied to the functions $f_{1}(z)=z \mathbf{v}$ and $f_{2}(z)=z^{2} \mathbf{v}$ yields

$$
\left(V_{T} \mathbf{v}\right)\left[\varphi_{T}(z)-\varphi_{T}(0)\right]=\left(V_{T}^{\prime} \mathbf{v}\right)\left[\psi_{T}(z)-\psi_{T}(0)\right]
$$

and

respectively. Then

$$
\left(V_{T} \mathbf{v}\right)\left[\varphi_{T}^{2}(z)-\varphi_{T}^{2}(0)\right]=\left(V_{T}^{\prime} \mathbf{v}\right)\left[\psi_{T}^{2}(z)-\psi_{T}^{2}(0)\right]
$$

$$
\begin{aligned}
{\left[\varphi_{T}(z)+\varphi_{T}(0)\right]\left(V_{T} \mathbf{v}\right)\left[\varphi_{T}(z)-\varphi_{T}(0)\right] } & =\left[\varphi_{T}(z)+\varphi_{T}(0)\right]\left(V_{T}^{\prime} \mathbf{v}\right)\left[\psi_{T}(z)-\psi_{T}(0)\right] \\
& =\left[\psi_{T}(z)+\psi_{T}(0)\right]\left(V_{T}^{\prime} \mathbf{v}\right)\left[\psi_{T}(z)-\psi_{T}(0)\right]
\end{aligned}
$$

implying that $\left[\varphi_{T}(z)+\varphi_{T}(0)\right]\left(V_{T}^{\prime} \mathbf{v}\right)=\left[\psi_{T}(z)+\psi_{T}(0)\right]\left(V_{T}^{\prime} \mathbf{v}\right)$, for every $z \neq 0$. Taking the inner product with $V_{T}^{\prime} \mathbf{v}$ and using the continuity of $\varphi_{T}$ and $\psi_{T}$ we have $\left[\varphi_{T}(z)+\varphi_{T}(0)\right]=\left[\psi_{T}(z)+\psi_{T}(0)\right]$, for every $z$. Then for $z=0$, we obtain that $\varphi_{T}(0)=\psi_{T}(0)$, then $\varphi_{T}(z)=\psi_{T}(z)$, for every $z \in \triangle$. These considerations imply that $\Phi$ is well defined. It is straightforward to show that $\Phi$ is one-to-one and onto. Now we show that $\Phi$ is a group isomorphism. It is clear from Theorem 3.1 the following:

$$
\begin{aligned}
(T S)(f)(z) & =U_{T S} f(0)+V_{T S}\left[f\left(\varphi_{T S}(z)\right)-f\left(\varphi_{T S}(0)\right)\right], \text { and } \\
(T S)(f)(z) & =U_{T}[\Phi(S)(f(0))]+V_{T}\left[\Phi(S)\left(f\left(\varphi_{T}(z)\right)\right)-\Phi(S)\left(f\left(\varphi_{T}(0)\right)\right)\right] \\
& =U_{T} U_{S} f(0)+V_{T} V_{S}\left[f\left(\left(\varphi_{S} \circ \varphi_{T}\right)(z)\right)-f\left(\left(\varphi_{S} \circ \varphi_{T}\right)(0)\right)\right] .
\end{aligned}
$$

The uniqueness of representation for the surjective isometries, shown above, implies that $U_{T S}=U_{T} U_{S}, V_{T S}=V_{T} V_{S}$ and $\varphi_{T S}=\varphi_{S} \circ \varphi_{T}$. Since $\Phi(T S)=$ $\left(U_{T S}, V_{T S}, \varphi_{T S}^{-1}\right), \Phi(T)=\left(U_{T}, V_{T}, \varphi_{T}^{-1}\right), \Phi(S)=\left(U_{S}, V_{S}, \varphi_{S}^{-1}\right)$, then $\Phi(T S)=$ $\Phi(T) \Phi(S)$. This proves that $\Phi$ is an isomorphism.

Corollary 3.3. If $G\left(\mathcal{S}_{\mathcal{H}}(\triangle)\right)$ is endowed with the strong operator topology and $\mathcal{U}(\mathcal{H}) \times \mathcal{U}(\mathcal{H}) \times A u t(\triangle)$ with the product of the strong operator topology on $\mathcal{U}(\mathcal{H})$ and the uniform topology on $\operatorname{Aut}(\triangle)$ then $G\left(\mathcal{S}_{\mathcal{H}}(\triangle)\right)$ and $\mathcal{U}(\mathcal{H}) \times \mathcal{U}(\mathcal{H}) \times \operatorname{Aut}(\triangle)$ are homeomorphic.

The proof of this corollary follow the same approach as the one used for Lemma 2.4 and thus it is omitted. We also observe that $G\left(\mathcal{S}_{\mathcal{H}}(\triangle)\right)$ is path connected. 


\section{Algebraic and Topological Structure of the Isometry Group of the little Bloch and Zygmund Spaces}

The little Bloch space consists of all analytic functions $f$ defined on $\triangle$, with values in a Banach space $E$, such that

$$
\lim _{|z| \rightarrow 1}\left(1-|z|^{2}\right)\left\|f^{\prime}(z)\right\|_{E}=0,
$$

where $\|\cdot\|_{E}$ is the norm on $E$. We refer the reader to the paper by Arregui and Blasco [2] for results on vector valued Bloch spaces. We will assume that $E$ is a smooth strictly convex and reflexive Banach space in order to apply a previous isometry theorem.

This space with the norm $\|f\|_{\mathcal{B}}=\|f(0)\|_{E}+\sup _{z \in \Delta}\left(1-|z|^{2}\right)\left\|f^{\prime}(z)\right\|_{E}$ is a Banach space, denoted by $\mathcal{B}_{*}(\triangle, E)$. We recall a characterization for the surjective isometries on this setting from [8].

Theorem 4.1. Let $E$ be a reflexive, smooth and strictly convex complex Banach space. Then $T: \mathcal{B}_{*}(\triangle, E) \rightarrow \mathcal{B}_{*}(\triangle, E)$ is a surjective linear isometry if and only if there exist surjective linear isometries $U, S: E \rightarrow E$ and a disc automorphism $\sigma$ such that for every $f \in \mathcal{B}_{*}(\triangle, E)$ and $z \in \triangle$,

$$
T(f)(z)=U f(0)+S[(f \circ \sigma)(x)-(f \circ \sigma)(0)] .
$$

The following corollary can be shown using previous arguments and thus the proof is omitted.

Corollary 4.2. Let $E$ be a reflexive, smooth and strictly convex complex Banach space. Then $G\left(\mathcal{B}_{*}(\triangle, E)\right)$ is isomorphic to the product $G(E) \times G(E) \times \operatorname{Aut}(\triangle)$.

We consider a vector valued version of the Zygmund space. We follow Blasco's definition in [6] for the vector valued Bloch space to introduce the vector valued Zygmund spaces. We determine the surjective isometries on the little Zygmund space and the algebraic structure of the isometry group.

The Zygmund space $\mathcal{Z}$ is the set of all analytic functions $f: \triangle \rightarrow E$ satisfying the boundedness condition

$$
\sup _{|z|<1}\left(1-|z|^{2}\right)\left\|f^{\prime \prime}(z)\right\|_{E}<\infty .
$$

This space endowed with the norm $\|f\|_{\mathcal{Z}}=\|f(0)\|_{E}+\left\|f^{\prime}(0)\right\|_{E}+\sup _{|z|<1}(1-$ $\left.|z|^{2}\right)\left\|f^{\prime \prime}(z)\right\|_{E}$ is a Banach space. We recall that the little Zygmund space is the closed subspace of $\mathcal{Z}$ defined by (see [?]):

$$
\mathcal{Z}_{0}=\left\{f \in \mathcal{Z}: \lim _{|z| \rightarrow 1^{-}}\left(1-|z|^{2}\right)\left\|f^{\prime \prime}(z)\right\|_{E}=0\right\} .
$$

Furthermore, we also consider the subspace of the little Zygmund space

$$
\mathcal{Z}_{0}^{(0,1)}=\left\{f \in \mathcal{Z}_{0}: f(0)=f^{\prime}(0)=0\right\} .
$$

We denote by $\mathcal{B}_{0}(\triangle, E)$ the subspace of the little Bloch space consisting of all functions that fix zero. It is clear that $\Phi: \mathcal{Z}_{0}^{(0,1)}(\triangle, E) \rightarrow \mathcal{B}_{0}(\triangle, E)$ defined by $\Phi(f)(z)=\int_{0}^{z} f(\xi) d \xi$ is a surjective isometry. Therefore given a surjective 
isometry $T$ on $\mathcal{Z}_{0}^{(0,1)}, \Phi T \Phi^{-1}$ is a surjective isometry on $\mathcal{B}_{0}(\triangle, E)$. Then we have the following characterization by using the form of the isometries on $\mathcal{B}_{*}(\triangle, E)$.

Theorem 4.3. Let $E$ be a reflexive, smooth and strictly convex complex Banach space then $T: \mathcal{Z}_{0}^{(0,1)}(\triangle, E) \rightarrow \mathcal{Z}_{0}^{(0,1)}(\triangle, E)$ is a surjective linear isometry if and only if there exist a disc automorphism $\sigma$ and a surjective isometry $V$ on $E$ such that for every $f \in \mathcal{Z}_{0}^{(0,1)}(\triangle, E)$ and $z \in \triangle$,

$$
\left.T f(z)=\int_{0}^{z} V\left[f^{\prime} \circ \sigma\right)(z)-\left(f^{\prime} \circ \sigma\right)(0)\right] d z .
$$

Following methods presented before we can show that $G\left(\mathcal{Z}_{0}^{(0,1)}\right)$ is isomorphic to the direct product $G(E) \times A u t(\triangle)$. For $\mathbf{v}$ a nonzero vector, we define $f_{1}(z)=\frac{z^{2}}{2} \mathbf{v}$ and $f_{2}(z)=\frac{z^{3}}{3} \mathbf{v}$. Then $\left(T f_{1}\right)^{\prime \prime}(z)=\sigma^{\prime}(z) V \mathbf{v}$, we select $\varphi \in E^{*}$ such that $\varphi(V \mathbf{v})=$ $\|\mathbf{v}\|_{E}$ thus $\varphi\left(\left(T f_{1}\right)^{\prime \prime}(z)\right) \neq 0$. On the other hand, $\left(T f_{2}\right)^{\prime \prime}(z)=2 \sigma(z)\left(T f_{1}\right)^{\prime \prime}(z)$ which implies that

$$
\sigma(z)=\frac{\varphi\left(\left(T f_{2}\right)^{\prime \prime}(z)\right)}{2 \varphi\left((T f)^{\prime \prime}(z)\right)}
$$

We conclude that $V \mathbf{v}=\frac{\left(T f_{1}\right)^{\prime \prime}(z)}{\sigma^{\prime}(z)}$. The previous considerations show that a surjective isometry $T$ determines $\sigma$ and $V$, the symbols appearing in the representation of $T$. We show that these symbols are uniquely defined. Suppose $V_{1}$ and $\sigma_{1}$ are additional symbols. Then for every $f$ and $z$, we have

$$
\left.\left.\int_{0}^{z} V\left[f^{\prime} \circ \sigma\right)(z)-\left(f^{\prime} \circ \sigma\right)(0)\right] d z=\int_{0}^{z} V_{1}\left[f^{\prime} \circ \sigma_{1}\right)(z)-\left(f^{\prime} \circ \sigma_{1}\right)(0)\right] d z .
$$

Considering $f_{1}$ and $f_{2}$ as defined above we have

$$
\left(T f_{1}\right)^{\prime}(z)=[\sigma(z)-\sigma(0)] V \mathbf{v}=\left[\sigma_{1}(z)-\sigma_{1}(0)\right] V_{1} \mathbf{v}
$$

and

$$
\left(T f_{2}\right)^{\prime \prime}(z)=\left[\sigma(z)^{2}-\sigma(0)^{2}\right] V \mathbf{v}=\left[\sigma_{1}(z)^{2}-\sigma_{1}\left(0^{2}\right)\right] V_{1} \mathbf{v}
$$

Therefore $\left[\sigma(z)^{2}-\sigma(0)^{2}\right] V \mathbf{v}=[\sigma(z)+\sigma(0)]\left[\sigma_{1}(z)-\sigma_{1}(0)\right] V_{1} \mathbf{v}=\left[\sigma_{1}(z)^{2}-\right.$ $\left.\sigma_{1}\left(0^{2}\right)\right] V_{1} \mathbf{v}$, and $\sigma(z)+\sigma(0)=\sigma_{1}(z)+\sigma_{1}(0)$ for every $z \in \triangle$. This implies that $\sigma=\sigma_{1}$ and $V=V_{1}$.

Then the mapping $\Phi(T)=(V, \sigma)$ is an isomorphism from $G\left(\mathcal{Z}_{0}^{(0,1)}(\triangle, E)\right)$ onto $G(E) \times \operatorname{Aut}(\triangle)$.

We observe that $\mathcal{Z}_{0}(\triangle, E)$ is isometric isomorphic to $E \oplus_{1} E \oplus_{1} \mathcal{Z}_{0}^{(0,1)}(\triangle, E)$, with norm $\|(u, v, f)\|=\|u\|_{E}+\|v\|_{E}+\sup _{|z|<1}\left(1-|z|^{2}\right)\left\|f^{\prime \prime}(z)\right\|_{E}$. Given $f \in$ $\mathcal{Z}_{0}(\triangle, E)$ we associate $\left(f(0), f^{\prime}(0), f_{0}\right) \in E \oplus_{1} E \oplus_{1} \mathcal{Z}_{0}^{(0,1)}(\triangle, E)$, with $f_{0}(z)=$ $f(z)-f(0)-f^{\prime}(0) z$. Hence a surjective isometry $T$ on $\mathcal{Z}_{0}(\triangle, E)$ induces a surjective isometry on $T_{0}$ on $\mathcal{Z}_{0}^{(0,1)}(\triangle, E)$ and an isometry on $E \oplus_{1} E$. For $f \in$ $\mathcal{Z}_{0}^{(0,1)}(\triangle, E)$, we set $T_{0}(f)(z)=(T f)(z)-(T f)(0)-(T f)^{\prime}(0) z$.

We now state a corollary describing all isometries on $\mathcal{Z}_{0}(\triangle, E)$ with the range space satisfying an additional property of not supporting any nontrivial $\ell_{1}$-projections, see [7]. 
Corollary 4.4. Let $E$ be a reflexive, smooth and strictly convex complex Banach space such that $E$ has no $\ell_{1}$ projections. Let $T: \mathcal{Z}_{0}(\triangle, E) \rightarrow \mathcal{Z}_{0}(\triangle, E)$, then $T$ is a surjective linear isometry if and only if there exist a disc automorphism $\sigma$, surjective isometries $U_{j}(j=1,2,3)$ on $E$ such that for every $f \in \mathcal{Z}_{0}(\triangle, E)$ and $z \in \triangle$,

or

$$
(T f)(z)=U_{1} f(0)+U_{2} f^{\prime}(0) z+\int_{0}^{z} U_{3}\left[f^{\prime}(\sigma(\xi))-f^{\prime}(\sigma(0))\right] d \xi
$$

$$
(T f)(z)=U_{1} f^{\prime}(0)+U_{2} f(0) z+\int_{0}^{z} U_{3}\left[f^{\prime}(\sigma(\xi))-f^{\prime}(\sigma(0))\right] d \xi .
$$

In the scalar case every isometry on $\mathcal{Z}_{0}(\triangle)$ is surjective, see [7], however this is no longer the case for $\mathcal{Z}_{0}(\triangle, E)$. Simply take $E=\ell_{2}$ and $U_{j}(j=1,2,3)$ to be a shift operator.

We now state a corollary that describes the structure of $G\left(\mathcal{Z}_{0}^{(0,1)}(\triangle, E)\right.$ ).

Corollary 4.5. Let $E$ be a reflexive, smooth and strictly convex complex Banach space such that $E$ has no $\ell_{1}$ projections. Then $G\left(\mathcal{Z}_{0}^{(0,1)}(\triangle, E)\right)$ is isomorphic to the direct product $\mathbb{Z}_{2} \times G(E) \times G(E) \times G(E) \times \operatorname{Aut}(\triangle)$.

We observe that for the scalar case $G\left(\mathcal{Z}_{0}^{(0,1)}\right)$ has two path connected components.

\section{Algebraic and Topological Structure of the Isometry Group OF $L^{p}$ SPACES}

In this section we consider vector valued $L^{p}$-spaces. Following the notation from [12], $X$ represents a separable Banach space, $(\Omega, \Sigma, \mu)$ is a measure space with $\Omega$ a nonempty set and $\Sigma$ the sigma-algebra of all $\mu$-measurable subsets of $\Omega$. The space $L^{\infty}(\mu, X)$ is the space of all essentially bounded measurable functions $f: \Omega \rightarrow X$ endowed with the $\|\cdot\|_{\infty}$, and $L^{p}(\mu, X)(1 \leq p<\infty, p \neq 2)$ is the space of all $p$-integrable functions $f: \Omega \rightarrow X$ endowed with the $p$-norm. We recall two important theorems that characterize the surjective linear isometries on these settings.

Theorem 5.1. (Greim) (cf. p. 70 in [12]) Let $X$ be a separable nonzero Banach space with trivial centralizer. Then each surjective isometry $U: L^{\infty}(\mu, X) \rightarrow$ $L^{\infty}(\mu, X)$ has the form

$$
U f(t)=V(t)(T f)(t), \text { for all } f \in L^{\infty}(\mu, X) \text { and } t \in \Omega,
$$

where $T$ is a regular set isomorphism on $\Sigma$, and $V$ is a strongly measurable operator-valued function such that for almost every $t \in \Omega, V(t)$ is a surjective isometry on $X$.

For the definition of a Banach space with trivial centralizer we refer the reader to Behrends book [4]. We also refer the reader to the books by Fleming and Jamison and references therein for the definitions of set isomorphism and strongly measurable operator-valued function (cf. [12] p. 55). 
The group of all isometries on $L^{\infty}(\mu, X)$ has two natural subgroups,

$$
G_{0}\left(L^{\infty}(\mu, X)\right)=\left\{U \in G\left(L^{\infty}(\mu, X)\right): U f(t)=V(t) f(t)\right\}
$$

and

$$
G_{1}\left(L^{\infty}(\mu, X)\right)=\left\{U \in G\left(L^{\infty}(\mu, X)\right): U f(t)=(T f)(t)\right\},
$$

where $V$ and $T$ are as described above.

It is straightforward to check that $G_{0}\left(L^{\infty}(\mu, X)\right)$ is isomorphic to $L^{0}(\mu, G(X))$ consisting of all strongly measurable functions $V: \Omega \rightarrow G(X)$, defined up to a set of $\mu$ measure zero. Also

$$
G_{1}\left(L^{\infty}(\mu, X)\right)=\left\{U \in G\left(L^{\infty}(\mu, X)\right): U f(t)=(T f)(t)\right\}
$$

is isomorphic to the group of all regular set isomorphisms $T$ on the sigma algebra; we denote such group by $A u t_{*}(\Sigma)$. We also denote by $T$ the map on $L^{\infty}(\mu, X)$ and induced by $T \chi_{A}=\chi_{T A}$ for every $A \in \Sigma$, as explained in [12].

Proposition 5.2. Let $X$ be a separable nonzero Banach space with trivial centralizer. Then the group of all surjective isometries on $L^{\infty}(\mu, X)$ is isomorphic to the semi-direct product $L^{0}(\mu, G(X)) \rtimes A u t_{*}(\Sigma)$.

Proof. We show that the representation of an isometry described in Theorem 5.1 is unique. Towards this fact we assume otherwise, then there exist strongly measurable functions and set isomorphisms such that

$$
V_{0}(t)\left(T_{0} f\right)(t)=V_{1}(t)\left(T_{1} f\right)(t), \quad \forall f \in L^{\infty}(\mu, X), \text { and } t \in \Omega .
$$

This last identity applied first to $f=\mathbf{v}$, a vector in $X$ and then to functions of the form $\chi_{A} \mathbf{v}$ with $A \in \Sigma$ yields $V_{0}(t) \mathbf{v}=V_{1}(t) \mathbf{v}$ for almost every $t$ and $T_{0} \chi_{A}=\chi_{T_{0} A}=\chi_{T_{1} A}=T_{1} \chi_{A}$. This implies that $V_{0}=V_{1}$ and $T_{0}=T_{1}$.

We observe that every isometry is the product of an isometry in $G_{0}\left(L^{\infty}(\mu, X)\right)$ with one in $G_{1}\left(L^{\infty}(\mu, X)\right)$. Furthermore, $G_{0}\left(L^{\infty}(\mu, X)\right)$ is a normal subgroup of $G\left(L^{\infty}(\mu, X)\right)$. Given $U \in G\left(L^{\infty}(\mu, X)\right)$ and $U_{0} \in G_{0}\left(L^{\infty}(\mu, X)\right)$ we have that $U^{-1} U_{0} U\left(\chi_{A} \mathbf{v}\right)(t)=\chi_{A}(t) V(t)^{-1} V_{0}(t) V(t) \mathbf{v}$, then extend to simple functions which are dense in $L^{\infty}(\mu, X)$. This implies that $U^{-1} U_{0} U \in G_{0}\left(L^{\infty}(\mu, X)\right)$. Also the intersection $G_{0}\left(L^{\infty}(\mu, X)\right) \cap G_{1}\left(L^{\infty}(\mu, X)\right)=\{I\}$. This completes the proof.

We now consider the case of isometries on $L^{p}(X, \mu)(1 \leq p<\infty$ and $p \neq 2)$. First we recall a characterization of the surjective isometries on this setting due to Sourour.

Theorem 5.3. (cf. p. 69 in [12]) Let $U$ be an operator on $L^{p}(\mu, X)$ with $1 \leq$ $p<\infty$ and $p \neq 2$ and such that the Banach space $X$ is separable complex and has the property that it is not the $\ell^{p}$ direct sum of two nonzero Banach spaces. Then $U$ is a surjective isometry if and only if

$$
U f(t)=h(t) V(t)(T f)(t), \text { for every } f \in L^{p}(\mu, X) \text { and } \mu-\text { a.e.t } \in \Omega,
$$

where $T$ is a set isomorphism from $\Sigma$ onto itself, $V \in L^{0}(\mu, \operatorname{Isom}(X))$ and $h=$ $\left(\frac{d \mu \circ T^{-1}}{d \mu}\right)$. 
We can now write a similar result given for $L^{p}$-spaces with $1 \leq p<\infty$ and $p \neq 2$. The proof is similar to the proof given for Proposition 5.2.

Proposition 5.4. Let $X$ a separable nonzero Banach space with the property that it is not the $\ell^{p}$ direct sum of two nonzero Banach spaces. Then the group of all surjective isometries on $L^{p}(\mu, X)$ is isomorphic to the semi-direct product $L^{0}(\mu, G(X)) \rtimes \operatorname{Aut}_{*}(\Sigma)$.

Acknowledgement. We are thankful to the referee for a very careful reading and suggestions for improvement.

\section{REFERENCES}

1. J. Arazy, Isometries of complex symmetric sequence spaces, Math. Z. 188 (1985), 427-431.

2. J. Arregui and O. Blasco, Bergman and Bloch spaces of vector valued functions, Math. Nachr. 261 (2003), 3-22.

3. S. Banach theorie des operations lineaires, Chelsea, Warsaw, 1932.

4. E. Behrends M-Structure and the Banach-Stone Theorem, Lecture Notes in Mathematics 736, Springer-Verlag, New York-Heidelberg, 1979.

5. E. Berkson and H. Porta, Hermitian operators and one parameter groups in Hardy spaces, Trans. Amer. Math. Soc. 185 (1973), 373-391.

6. O. Blasco, Operators on weighted Bergman spaces $(0<p \leq 1)$ and applications, Duke math. J. 66:3 (1992), 443-467.

7. F. Botelho, Isometries and Hermitian Operators on Zygmund spaces, Canad. Bull. Math. (to appear), doi: 10.4153/CMB-2014-071-5.

8. F. Botelho and J. Jamison, Isometries on the vector valued little Bloch space, Illinois J. Math., to appear.

9. M. Cambern, The isometries of $H^{\infty}(K)$, Proc. Amer. Math. Soc. 36 (1972), 173-178.

10. M. Cambern and K. Jarosz, The isometries of $H_{\mathcal{H}}^{1}$, Proc. Amer. Math. Soc. 107 (1989), no. 1, 205-214.

11. D.S. Dummit and R.M. Foote, Abstract Algebra, John Wiley \& Sons, Inc., Hoboken, N.J., 2004.

12. R. Fleming and J. Jamison Isometries on Banach Spaces:Vector-Valued Function Spaces, Chapman \& Hall/CRC, Boca Raton, 2008.

13. F. Forelli The isometries of $H^{p}$, Canad. J. Math. 16 (1964) 721-728.

14. E. Gardella and H. Thiel Banach algebras generated by an invertible isometry of an $L^{p}$ space, arXiv:1405.5589v1.

15. W. Hornor and J.E. Jamison Isometries of some Banach spaces of analytic functions, Integral Equations Operator Theory 41 (2001), 410-425.

16. P. Koszmider, M. Martín and J. Merí Extremely non-complex $C(K)$ spaces, Cent. Eur. J. Math. 9 (2011), no. 4, 797-802.

17. P. Koszmider, M. Martín and J. Merí Isometries on extremely non-complex Banach spaces, J. Inst. Math. Jussieu 10 (2011), no. 2, 325-348.

18. K. Jarosz Any Banach space has an equivalent norm with trivial isometries, Israel J. Math 64 (1988), no. 1, 49-56.

19. P.-K. Lin The isometries of $H^{\infty}(E)$ Pacific J. Math. 143 (1990), no. 1, 69-77.

20. P.-K. Lin The isometries of $H^{p}(K)$, J. Austral. Math. Soc. Ser. A 50 (1991), no. 1, 23-33.

Department of Mathematical Sciences, The University of Memphis, Memphis, TN 38152, USA.

E-mail address: mbotelho@memphis.edu

E-mail address: jjamison@memphis.edu 